\title{
Metrology for MRI Safety
}

\author{
Bernd Ittermann ${ }^{1, a}$, Oriano Bottauscio ${ }^{2}$, Jeffrey Hand ${ }^{3}$, Jacco de Pooter ${ }^{4}$, Leon de Prez ${ }^{4}$, Hans Rabus ${ }^{1}$, Frank Seifert ${ }^{1}$, \\ Hanitra Szymanowski ${ }^{1}$, Gerd Weidemann ${ }^{1}$ and Luca Zilberti $^{2}$ \\ ${ }^{1}$ Physikalisch-Technische Bundesanstalt (PTB), Braunschweig and Berlin, Germany \\ ${ }^{2}$ Istituto Nazionale di Ricerca Metrologica, Torino, Italy \\ ${ }^{3}$ Division of Imaging Sciences and Biomedical Engineering, King's College London, London, United Kingdom \\ ${ }^{4}$ VSL, Delft, The Netherlands
}

\begin{abstract}
Magnetic Resonance Imaging (MRI) has become an indispensable medical imaging modality with about 30 million patient exams in the EU every year and an excellent history of safe use. Nevertheless, it is continuously evolving and recent technological developments such as ultrahigh magnetic fields, parallel transmission, or MRI guided radiotherapy promise to significantly enhance the quality and the range of applicability of MRI. A major reason why these technological developments are not yet used in the clinical practice are unresolved safety issues. If the patient risk cannot be quantified reliably, a 'safety first' attitude naturally prevails preventing the routine use of new technologies or the scanning of subjects at high risk, e.g. carriers of metallic medical implants. The EMRP joint research project HLT 06 "Metrology for MRI Safety" aimed at providing such risk assessments for certain new developments or applications in MRI. The project was concluded in 2015 and some key results will be presented here.
\end{abstract}

\section{Introduction}

MRI is one of most important medical imaging modalities today. It is an indispensable tool in diagnosis and therapy control of neurologic, oncologic, cardiovascular, or musculoskeletal diseases. Its adjustable contrast capabilities particularly with respect to soft tissue are unmatched and cannot be provided by any other imaging modality. Worldwide, about 100 million MRI scans are performed per year. OECD statistics indicate about $4 \%$ of the western and $2.5 \%$ of the eastern European population having an MRI scan every year, numbers growing by $10 \%$ p.a. in Western and $20 \%$ p.a. in Eastern Europe. Every matter affecting safety, performance or the applicability of MRI has an impact on European society as a whole, therefore. The fast implementation of innovative technological developments into the clinical practice is frequently impeded by unsolved safety issues. There is a prevalent uncertainty about how to ensure patient safety if new developments like parallel transmission come into play or carriers of medical implants are to be scanned. The current project aimed to specifically address these issues.

Another exciting new development is the MRIaccelerator combination. This emerging technology fuses MRI and radiotherapy and provides precise, soft-tissue based, on-line position verification and treatment monitoring during external-beam tumour therapy. This has the potential to revolutionise cancer treatment but as long as a traceable gamma dosimetry inside an MRI scanner was completely missing, any clinical application of the new technology was prohibitive. To close this gap was another major goal of the described research project.

\section{The project objectives}

\footnotetext{
${ }^{\text {a } C o r r e s p o n d i n g ~ a u t h o r: ~ b e r n d . i t t e r m a n n @ p t b . d e ~}$
}

The specific project objectives were:

i) to produce reference instrumentation for traceable measurements of RF electromagnetic fields generated by MRI scanners; these measurements should be suitable to validate the modelling results;

ii) to develop a mathematical modelling concept to calculate RF electromagnetic field distributions inside the human body;

iii) to develop an assessment of the risk to human subjects from moving through the inhomogeneous stray fields of MRI scanners which can induce eddy currents in the $1-\mathrm{Hz}$ regime inside the human body;

$i v)$ to develop a validated measurement method for the assessment of Specific Absorption Rate (SAR) hazards associated with emerging new MRI technologies like parallel transmission (pTx) or ultrahigh magnetic fields;

$v$ ) to develop a dosimetry method for the application of MRI guided radiotherapy, comprising a system for traceably measuring the absorbed dose to water for high energy photon beams in an MRI-accelerator combination and assessment of the potential changes in relative biological effectiveness of photon beams due to the magnetic field;

vi) to develop a method for the assessment of the risks due to the presence of passive, metallic, medical implants inside the patient's body during an MRI scan.

\section{Results}

\subsection{RF electromagnetic field measurements}

This is an Open Access article distributed under the terms of the Creative Commons Attribution License 4.0, which permits unrestricted use, distribution, and reproduction in any medium, provided the original work is properly cited. 
Within the work on RF electromagnetic field measurements an MR compatible TEM cell was designed and built [1]. Simulations and in-situ measurements show that this device allows traceable in situ calibrations of RF field probes right in the bore of an MR scanner. For current measurements a body phantom with a phantom liquid characterised at $64 \mathrm{MHz}, 128 \mathrm{MHz}$, and $300 \mathrm{MHz}$ and a gantry for reproducible positioning of field probes was applied. Using these developments extensive calibrated field measurements in and around a body phantom inside a 3T MRI scanner were performed and successfully employed to evaluate the accuracy of simulations. Those measurements were then extended to a human subject in the scanner. Field profiles were acquired when the sensor was moved along linear paths above the subject. Comparison to numerical simulations on a human voxel model showed good agreement, thus proving the usefulness of this approach but also the necessity of an extensive phantom based optimisation process which had preceded the in vivo measurements. While this instrumentation was primarily developed for use within the project it had also been take up by end users. Stakeholders from academia who were aware of the new measurement capabilities actively sought cooperation with project partners for an ongoing applied research project on safety issues of coronary stents in cardiac MRI [2].
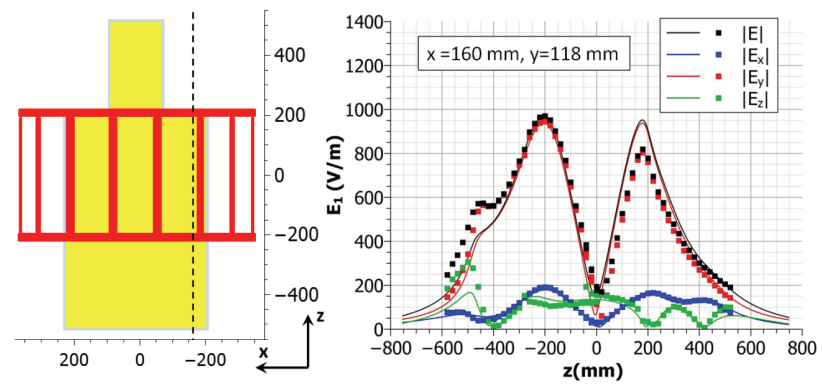

Figure 1. Left: Body phantom filled with tissue simulating liquid within the 'body coil' RF transmitter of a clinical 3T scanner. Right: RF E-field components measured (dots) with field probes along the indicated linear path above the phantom and corresponding simulations (lines).

\subsection{RF electromagnetic field calculations}

Various types of EM field measurements have been compared to simulations and this verifies how detailed the coil model is required to be. A frequency domain hybrid solver, combining aspects of the Finite Element Method (FEM) and the Boundary Elements Method (BEM) was developed [3] and applied to analyze the sensitivity of external fields to internally induced phenomena in phantoms. An extensive inter-comparison of electromagnetic codes was completed and the results compared to those obtained through experiments on a phantom which had been specifically developed for this purpose. The analysis was then extended to anatomical voxel models.

The simulation of the exposure of human voxel models to MRI electromagnetic fields was then extended to include thermal effects. The most important result is a rather poor spatial correlation between the maximum temperature change and local SAR, thus questioning traditional SAR based safety concepts which are, at present, the state-of-the-art in all relevant standards [4]. These findings are in agreement with observations from other researchers and are already being discussed by the relevant standards bodies. Short term solutions are not in reach, however, as so far no means exist to check and validate the thermal simulations.
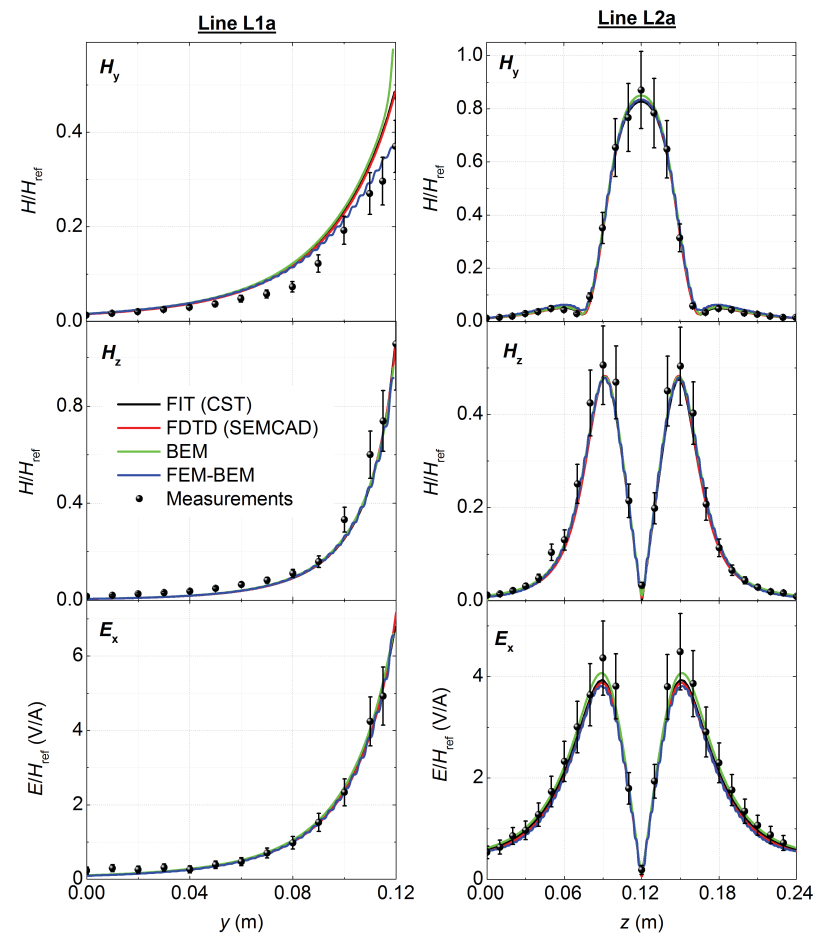

Figure 2. Comparison among measured and computed normalized RF field components $\left(H_{\mathrm{y}}, H_{\mathrm{z}}, E_{\mathrm{x}}\right)$ along two different paths (L1a, L2a) within an irradiated phantom at $64 \mathrm{MHz}$. The different lines represent four different numerical algorithms.

\subsection{Motion induced low frequency fields and currents}

To investigate motion induced low-frequency fields, e.g. a human subject moving in the fringe field of an MRI magnet, a Green's function based BEM algorithm [5] was implemented. One peculiarity of this approach is that it was completely developed in the reference frame of the human body rather than in the laboratory frame. This provides robust behaviour even for body rotations, a type of motion known to need careful inspection with the more widespread potential-based modelling techniques. It could be shown that this BEM approach is intrinsically better adopted, compared to alternative methods, to calculate the extremely low-frequency electric fields inside the human body induced by movements in stray magnetic fields. The consistency of the BEM approach, which had been questioned in the literature [6], was convincingly demonstrated [7]. A new FEM formulation, developed and implemented in a parallelised computational code suitable to simulate motion-induced fields in 
high resolution anatomical models, represented the next step forward, as it utilized the experience gained with the FEM-BEM code developed for RF problems. The new formulation is capable to describe the contribution of both conduction and dielectric currents. The FEM code which was found to be very adequate for studying high resolution anatomical models was exploited to analyse effects introduced by dielectric currents, the dispersion of tissue parameters and, most importantly, accelerated/decelerated motion phases on the induced electric field [8]. Based on this analysis a set of simulations describing the exposure under realistic conditions was derived.

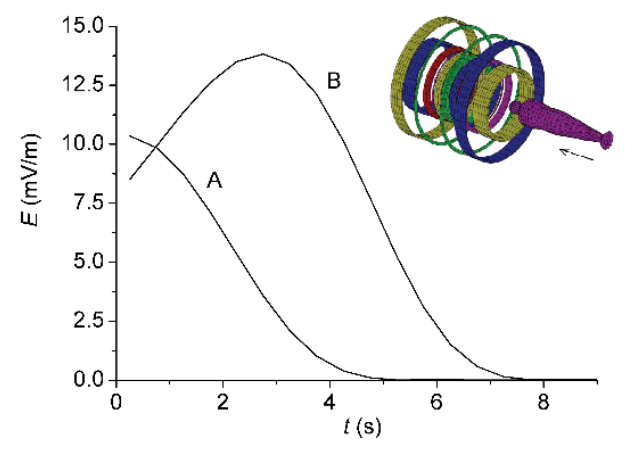

Figure 3. Example of computed motion-induced $E$-fields in two different locations of a simplified model of the human body (A: head, B: shoulder) entering a $1.5 \mathrm{~T}$ scanner with a uniform speed of $0.1 \mathrm{~m} / \mathrm{s}$.

Stakeholders from an academic research group investigating the feasibility of a new MRI-accelerator design with the patient rotating within the scanner took up these results and asked for co-operation to utilize JRP expertise for their specific application. This collaboration turned out to be very fruitful $[9,10]$ and provides external links to the project, e.g. on motion induced LF fields and on the MRI accelerator, which had not initially been anticipated.

In response to external developments, i.e. a new edition of ICNIRP guidelines [11] and feedback from reviewers, the scope of the work on motion induced fields was adapted. Both the maximum variation of magnetic flux density vector over a given time interval (to prevent from vertigo and nausea when moving within the stray field of an MRI magnet) and the signal of the induced electric field scaled through the weighted-peak approach (to prevent from magneto-phosphenes and peripheral nerve stimulation) were evaluated as proposed by the new guidelines. One finding was that different mathematical approaches, deemed equivalent in the guidelines, can produce strikingly different results. This stresses the necessity for a revision of the guidelines in this respect.

\subsection{Emerging technologies: pTx and ultrahigh fields}

A setup for in-situ measurements of complete Sparameter matrices for parallel transmit systems in MRI was designed and assembled. The completed device was successfully tested and its first application was the in-situ measurement of S-parameter matrices of an 8-channel coil under different loading conditions [12]. These Sparameter measurements allowed the partners to determine the absorbed RF power of the system coil/object for all steering conditions. The new measurement system is specifically designed to work with any pTx-capable MRI scanner, i.e. independent of vendor specific hardware or software. The ultimate goal of the S-parameter measurement system is a complete assessment of the power balance of a given pTx coil and its auxiliary hardware.

For 7T-MRI, power losses by radiation to the far field can no longer be assumed to be negligible. A procedure for the detection of radiated power was developed using a $60 \mathrm{~cm}$ copper clad tunnel mimicking the bore of a 7T magnet. Since the signals are small the measurements have to be done in a carefully shielded environment. First results for a commercial 8-channel loop array show that the circularly polarised excitation mode radiates the most power. This is in perfect agreement with recently published numerical results [13].

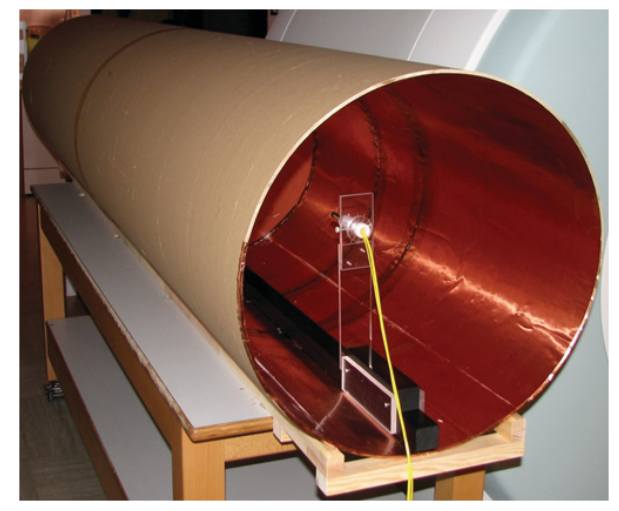

Figure 4. Setup with a time-domain opto-electric field sensor for measuring the radiated RF power from transmit coils for 7T MRI.

\subsection{Emerging technologies: MRI-accelerator}

In late 2012 two leading European manufacturers of radiation therapy accelerators and MRI scanners, respectively, announced that they would take their MRIaccelerator project to the next level and expand the existing research collaboration with University Medical Center (UMC) Utrecht to a worldwide collaboration network. A new water calorimeter suitable for operation in high magnetic fields which was successfully designed and built within the 'MRI Safety' project had been an indispensable prerequisite for this strategic decision. Monte Carlo simulations demonstrated the capability of this new calorimeter to achieve the required thermal stability and meanwhile the device was successfully commissioned and tested. 
This highly versatile calorimeter will be used as a new primary standard for absorbed dose to water in highenergy photon beams and medium-energy x-rays. Considerable effort was taken to make the device as compact as possible and to make it transportable. It will be brought directly to the end users at UMC Utrecht, and later possibly to other sites, as well, and will be used in the high magnetic field of their MRI-accelerator system. Due to its design and material choices the calorimeter will be applicable in a wide variety of beam modalities such as photons, electrons, protons and heavy ion-beams. The conservation of radiation in the calorimeter was studied by simulations [12] and the validation of the new calorimeter was a major milestone. First this was done in the VSL ${ }^{60} \mathrm{Co}$ beam, then on-site at Netherlands Cancer Institute AVL in Amsterdam in $6 \mathrm{MV}$ and $10 \mathrm{MV}$ clinical photon beams (flattening and non-flattening filter), and eventually it took part in the key comparison BIPM.RI(I)$\mathrm{K} 6$ at NPL in photon beams of $6 \mathrm{MV}, 10 \mathrm{MV}$ and 25 MV. The new water calorimeter will be fully commissioned as a primary standard for MV-photon beams after the results have been released. It will become an internationally accepted primary standard that is also capable of establishing the absorbed-dose-to-water in the presence of a strong magnetic field.

To investigate potentially altered mechanisms of biological radiation damage in magnetic fields the triple differential cross sections (TDCS) of water for electron scattering were calculated. The results showed that the TDCS data in condensed history simulation codes for radiation transport do not need to be modified while simulating macroscopic dose distributions in the presence of magnetic fields. On a nanoscopic scale, in contrast, the variation of the TDCS with the orientation of the water molecules may affect the particle track structure and thus needs to be accounted for. A computer programme for simulation of the track structure of electrons with energy between $40 \mathrm{eV}$ and $1 \mathrm{keV}$ was thus developed which accounts for the effect of the polarised water molecules on the electron scattering cross sections.

\subsection{Metallic implants in MRI}

New mathematical and numerical tools were developed for advanced RF electro-magnetic field simulations and detailed human voxel models containing a hip prosthesis were constructed $[10,15]$. This enabled the project partners to assess MRI safety issues related to metallic medical implants. SAR distributions around implanted hip prostheses were investigated for a variety of subject positions within an MRI scanner and a worst-case position was identified. This approach was then extended to include both EM and thermal simulations of anatomical models in the presence of orthopaedic metallic implants, also in view of possible external field monitoring. First activities were also started regarding the investigation on wire implants, beginning with an analysis of the modelling issues caused by the presence of thin and long metallic structures within anatomical human models.
A calibrated current sensor was built and successfully tested, suitable to detect RF induced currents in wire-like implants if they protrude from the body. Towards the end of the project this sensor was applied in phantom studies to validate the corresponding simulation results. Albeit not yet tested, the sensor, as it is applied externally, can in principle be used on patients, too, if they are carrying similarly protruding devices, e.g. deep brain stimulator electrodes.
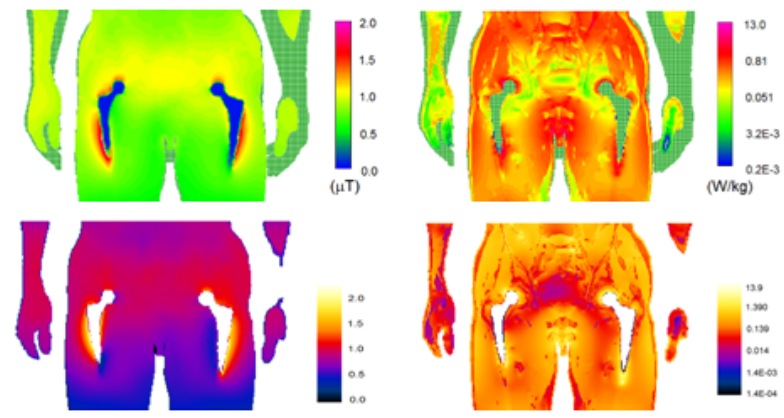

Figure 5. Comparison of simulated maps of the RF magnetic field component $B_{1}{ }^{+}$(left), and local SAR (right, on a logarithmic scale) in an anatomical human voxel with a bilateral hip prosthesis exposed to RF from the body coil of a 3T MRI scanner. Top row: self developed FEM/BEM hybrid code. Bottom row: commercial FDTD code (SEMCAD-X).

\section{Conclusion}

The major long term impacts aimed at by this JRP had been: $i$ ) financial savings for the EU health care systems due to reduced MRI scan times, ii) social advancements by helping to make MRI accessible to implant carriers, and iii) social advancements via improved cancer treatment by the MRI-accelerator. Clearly, it was neither possible nor intended to achieve these ultimate objectives within the project lifetime. Nevertheless, significant advancements towards these goals could be achieved. The assessment of the MRI safety of certain generic implant types was improved. This will assist manufacturers developing "MR safe" implants and will also reduce the time spent by clinicians to assess the MRI safety of implant carrying patients. Simulation tools to assess the interaction of time varying EM fields with metallic implants were validated by comparison with measurements and the scientific and metrological uncertainties in these areas were reduced. The MRI accelerator advanced from a proof-of-principle laboratory setup to a professionally manufactured clinical prototype soon to be delivered to top-level research hospitals and JRP results contributed their share to this success story.

In summary, the multinational cooperation effort with critical contributions from all partners paid off and allowed a much wider and farther-reaching approach towards MRI safety compared to what any single participating group could have attempted. All specific project objectives as stated in the introduction of this paper were achieved.

\section{Acknowledgements}


This work was supported by EMRP grant HLT06. The EMRP is jointly funded by the EMRP participating countries within EURAMET and the European Union.

\section{References}

1. T. Klepsch, T.D. Lindel, W. Hoffmann, H. Botterweck, B. Ittermann, F. Seifert, Biomed. Tech. 57 Suppl. 1, 119 (2012)

2. L. Winter, E. Oberacker, C. Ozerdem, Y. Ji, F. von Knobelsdorff-Brenkenhoff, G. Weidemann, B. Ittermann, F. Seifert, T. Niendorf, Magn. Reson. Med. (2014). doi: 10.1002/mrm.25483 [Epub]

3. O. Bottauscio, M. Chiampi, L. Zilberti, Bioelectromagn. 49, 15-21 (2014)

4. IEC 60601-2-33 Ed. 3 (2010)

5. M. Chiampi, L. Zilberti, IEEE Trans Biomed Eng. 58, 2787 (2011)

6. C.C. Sanchez, IEEE Trans. Biomed. Eng. 60, 880 (2013)

7. M. Chiampi, L. Zilberti, IEEE Trans. Biomed. Eng., 60, $882(2013)$

8. L. Zilberti, O. Bottauscio, M. Chiampi, IEEE Magnetics Letters 6, 1500104 (2015)

9. A. Trakic, L. Liu, H. Sanchez Lopez, L. Zilberti, F. Liu, S. Crozier, IEEE Trans. Biomed. Eng. 61, 784 (2014)

10. L. Zilberti, O. Bottauscio, M. Chiampi, J. Hand, H. Sanchez Lopez, R. Brühl, S. Crozier, Magn. Reson. Med. (2015) doi: 10.1002/mrm.25687 [Epub]

11. ICNIRP, Health Phys. 106, 418 (2014)

12. G. Weidemann, F. Seifert, W. Hoffmann, R. Seemann, P. Waxmann B. Ittermann, Proc. ISMRM 23, 1775 (2015)

13. A Kuehne, S Goluch, P Waxmann, F Seifert, B Ittermann, E Moser, E Laistler, Magn Reson Med. (2014) doi: 10.1002/mrm.25493 [Epub]

14. J.A de Pooter, L.A. de Prez, H. Bouchard, Phys. Med. Biol. (2015), in press

15. A. Papadaki, J. Hand, J. Powell, D. McRobbie, A. Hart, Proc. ISMRM 20, 1416 (2012)

16. G. Weidemann, F. Seifert, W. Hoffmann, B. Ittermann, Proc. ISMRM 23, 1852 (2015) 